\title{
MAPPING CLASS GROUPS ARE AUTOMATIC
}

\author{
LeE Mosher
}

Let $S$ be a compact surface, possibly with the extra structure of an orientation or a finite set of distinguished points called punctures. The mapping class group of $S$ is the group $\mathcal{M C G}(S)=\operatorname{Homeo}(S) / \operatorname{Homeo}_{0}(S)$, where $\operatorname{Homeo}(S)$ is the group of all homeomorphisms of $S$ preserving the extra structure, and $\mathrm{Homeo}_{0}(S)$ is the normal subgroup of all homeomorphisms isotopic to the identity through elements of $\operatorname{Homeo}(S)$. By convention each boundary component of $S$ contains a puncture; in general, if a boundary component contains no puncture it may be collapsed to a puncture without changing the mapping class group.

Given a group $G$, suppose that $A=\left\{g_{1}, \ldots, g_{k}\right\}$ is a finite set of generators and $L$ is a set of words over the alphabet $A$, such that each element of $G$ is represented by at least one word in $L$, and $L$ is a regular language over $A$, i.e. one can check for membership in $L$ with a finite automaton. The words in $L$ representing a given group element can be thought of as normal forms for that element. Then $L$ is an automatic structure for $G$ if for any two words $v, w \in L$, one can check with a finite automaton whether the associated group elements $\bar{v}$ and $\bar{w}$ are equal, and whether they differ by a certain generator. A more geometric characterization of automaticity is given by the fellow traveller property, which says that there is a constant $K$ such that for any $v, w \in L$, if $d(\bar{v}, \bar{w}) \leq 1$, where $d(\bar{v}, \bar{w})$ is the word length of $\bar{v}^{-1} \bar{w}$, then for any $n \geq 0$, letting $v(n)$ and $w(n)$ be the prefixes of $\bar{v}$ and $\bar{w}$ of length $n$, then $d(\bar{v}(n), \bar{w}(n)) \leq K$. A group $G$ is automatic if it has an automatic structure. The theory of automatic groups is presented in [ECHLPT]. An automatic group has a quadratic isoperimetric inequality, and a quadratic time algorithm for the word problem, in addition to many other nice geometric and computational properties.

Theorem [M3]. The group $\mathcal{M C G}(S)$ is automatic.

In $[\mathrm{P}]$, Penner computes the action of $\mathcal{M C G}(S)$ on the Dehn-Thurston parameterization of the space of simple closed curves on $S$. An algorithm

Received November 28, 1993.

The author was partially supported by NSF grant \#DMS-9204331. 
for the word problem in $\mathcal{M C G}(S)$ may be derived from this computation: given a word in the generators, carry out Penner's computation and see if the action is trivial. When $S$ is a punctured surface a similar algorithm may be described using ideal triangulations and elementary moves (see [M2] for the necessary computations), and the resulting formulas are even simpler than in Penner's computation. This leads one to wonder whether ideal triangulations and elementary moves can be used to give an automatic structure when $S$ is punctured, and this is what we do. The main idea is to "comb" one ideal triangulation along the arcs of another, in a manner similar to that used by Hatcher $[\mathrm{H}]$ to prove contractibility of the ideal arc complex of $S$. When $S$ is a closed surface, we were not able to convert Penner's algorithm into an automatic structure; we have found an automatic structure using other ideas.

The proofs use results on groupoids described in [ECHLPT section 11.1]. Suppose $\Gamma$ is a groupoid, whose objects are called vertices. The morphisms of $\Gamma$ with initial vertex $x$ are denoted $\Gamma_{x}$, those with terminal vertex $y$ are denoted $\Gamma^{y}$, and $\Gamma_{x}^{y}=\Gamma_{x} \cap \Gamma^{y}$. Associated to each vertex $x$ of $\Gamma$ is the group $\Gamma_{x}^{x}$, which is well-defined up to isomorphism independent of $x$. An automatic structure for $\Gamma$ is defined by choosing a base vertex $v$, a generating set $A=\left\{g_{1}, \ldots, g_{k}\right\}$ of morphisms, and a regular language $L$ over $A$ representing each morphism of $\Gamma_{v}$ at least once, such that the fellow traveller property is satisfied. Then [ECHLPT, theorem 11.1.3] says that the associated group $\Gamma_{v}^{v}$ is automatic if and only if the groupoid $\Gamma$ is automatic. We shall describe an automatic groupoid whose associated group is $\mathcal{M C G}(S)$.

First we do the case where $S$ is a punctured surface, and for simplicity we assume $S$ is oriented. An ideal triangulation of $S$ is a triangulation whose vertex set is the set of punctures. The edges of the triangulation are called ideal arcs. In general, if an ideal triangulation is labelled by choosing an oriented ideal arc, then the only elements of $\operatorname{Homeo}(S)$ which preserve the triangulation and the labelling are elements in $\operatorname{Homeo}_{0}(S)$. Thus, by convention we insist that each ideal triangulation be labelled. Let $\mathcal{D}(S)$ be the set of isotopy classes of ideal triangulations on $S$. Then $\mathcal{M C G}(S)$ acts freely on $\mathcal{D}(S)$, and it follows by general principles that orbits of the diagonal action of $\mathcal{M C G}(S)$ on $\mathcal{D}(S) \times \mathcal{D}(S)$ form a groupoid whose associated group is $\mathcal{M C G}(S)$; we call this the mapping class groupoid of $S$, denoted $\mathcal{M C G D}(S)$. The vertices of $\mathcal{M C G D}(S)$ are the orbits of the action of $\mathcal{M C G}(S)$ on $\mathcal{D}(S)$. The vertex corresponding to an ideal triangulation $\delta$ is denoted $\{\delta\}$, and the morphism corresponding to an ordered pair of ideal triangulations $\left(\delta, \delta^{\prime}\right)$ is denoted $\left\{\delta, \delta^{\prime}\right\}$.

The groupoid $\mathcal{M C G D}(S)$ is generated by two types of morphisms. If 
$\delta$ and $\delta^{\prime}$ differ only by a change of labelling, then the morphism $\left\{\delta, \delta^{\prime}\right\}$ is called a relabelling generator. To define the second type of generator, consider an ideal triangulation $\delta$ and an ideal arc $h$ of $\delta$. If there are two distinct triangles on either side of $h$, then $h$ may be removed and the two triangles joined into a quadrilateral $Q$, and the opposite diagonal $h^{\prime}$ of $Q$ may be inserted to form another ideal triangulation $\delta^{\prime}$. We say that $\delta \rightarrow \delta^{\prime}$ is an elementary move performed on $h$, with support $Q$. The resulting morphism denoted $\left\{\delta \rightarrow \delta^{\prime}\right\}$ is called an elementary move generator. The set of all relabelling and elementary move generators forms a symmetric generating set for $\mathcal{M C G D}(S)$ denoted $A_{0}$.

As a first step, we construct an asynchronous automatic structure $L_{0}$ over the alphabet $A_{0}$ for $\mathcal{M C G D}(S)$. This means that the fellow traveller property is replaced by the weaker "asynchronous fellow traveller property": there is a constant $K$ such that for each $v, w \in L_{0}$, if $d(\bar{v}, \bar{w}) \leq 1$ then there are strictly increasing subsequences $v\left(n_{i}\right), w\left(m_{i}\right)$ with $n_{0}=m_{0}=0$, $m_{i+1}-m_{i} \leq K, n_{i+1}-n_{i} \leq K$, and $d\left(\bar{v}\left(n_{i}\right), \bar{w}\left(m_{i}\right)\right) \leq K$ for all $i$.

Fix a base ideal triangulation $\delta_{B}$, so $\left\{\delta_{B}\right\}$ will be the base vertex for $\mathcal{M C G D}(S)$. Fix once and for all an orientation for each ideal arc of $\delta_{B}$, and choose an enumeration $\left\{g_{1}, \ldots, g_{n}\right\}$ of the ideal arcs of $\delta_{B}$. Given $\delta$, a normal form for the morphism $\left\{\delta_{B}, \delta\right\}$ is obtained by "combing" $\delta$ along the arcs of $\delta_{B}$. First isotop so that $\delta$ and $\delta_{B}$ are pulled tight with respect to each other. Some arcs of $\delta_{B}$ may then be identical to arcs of $\delta$; we say that $\delta$ is combed along these arcs. Let $g_{i}$ be the first arc of $\delta_{B}$ along which $\delta$ is uncombed. The arc $g_{i}$ emerges from some corner (called a prong) of some triangle $T$ of $\delta$, traverses $T$ to the opposite edge $h$, and then crosses $h$ into the next triangle. There is an elementary move $\delta \rightarrow \delta^{\prime}$ performed on $h$, called the first step in combing $\delta$ along $\delta_{B}$. Now define a sequence of elementary moves $\delta=\delta_{0} \rightarrow \delta_{1} \rightarrow \cdots$ such that for each $n \geq 0, \delta_{n} \rightarrow \delta_{n+1}$ is the first step of combing $\delta_{n}$ along $\delta_{B}$. One checks that this sequence terminates in an ideal triangulation $\delta_{N}$ which is a relabelling of $\delta_{B}$, and $\delta_{0} \rightarrow \cdots \rightarrow \delta_{N}$ is called the combing sequence of $\delta$. The normal form for the morphism $\left\{\delta_{B}, \delta_{0}\right\}$ is taken to be $w_{0}(\delta)=$ $\left\{\delta_{B}, \delta_{N}\right\} \circ\left\{\delta_{N} \rightarrow \delta_{N-1}\right\} \circ \cdots \circ\left\{\delta_{1} \rightarrow \delta_{0}\right\}$, a single relabelling generator followed by a sequence of elementary move generators. By construction, each morphism in $\mathcal{M C G D}(S)$ has a unique normal form, which defines $L_{0}$. Regularity of $L_{0}$ is proved by constructing an automaton that remembers three pieces of data: the "combinatorial type" of $\delta$, the ideal arcs of $\delta_{B}$ along which $\delta$ is combed, and the prongs of $\delta$ from which the remaining arcs of $\delta_{B}$ emerge.

Theorem. The language $L_{0}$ is an asynchronous automatic structure for $\mathcal{M C G D}(S)$. 
This result was discovered previously by Hatcher and Vogtmann [HV].

The theorem is proved by applying relators to sequences of elementary moves, using techniques very much like those presented in [M1] for computing conjugacy invariants of pseudo-Anosov elements of $\mathcal{M C G}(S)$. Defining relators for $\mathcal{M C G D}(S)$ are constructed as follows. Given two elementary moves $\delta \rightarrow \delta^{\prime}, \delta \rightarrow \delta^{\prime \prime}$ performed on $h, h^{\prime}$ respectively, if the supports are disjoint then the elementary moves commute, meaning that there are elementary moves $\delta^{\prime} \rightarrow \delta^{\prime \prime \prime}$ and $\delta^{\prime \prime} \rightarrow \delta^{\prime \prime \prime}$ performed on $h^{\prime}, h$ respectively; this forms a relator of four elementary moves called a commutator relator. On the other hand, the supports may overlap in a single triangle, hence their union is a pentagon, and there is a sequence of five elementary moves supported in this pentagon, called a pentagon relator. Suppose that $\delta_{0} \rightarrow \cdots \rightarrow \delta_{N}$ is the combing sequence for $\delta_{0}$, and $\delta_{0} \rightarrow \delta_{0}^{\prime}$ is an elementary move. It may happen that this elementary move extends or reduces the normal form by a single elementary move, in which case the fellow traveller property with $K=1$ is immediate. If this does not happen, let $\delta_{0}^{\prime} \rightarrow \cdots \rightarrow \delta_{N^{\prime}}^{\prime}$ be the combing sequence for $\delta_{0}^{\prime}$. Doing a case by case analysis, applying either a commutator relator or a pentagon relator, one proves either that there is an elementary move $\delta_{i} \rightarrow \delta_{j}^{\prime}$ with $(i, j) \in\{(1,1),(1,2),(2,1)\}$, or that $\delta_{2}=\delta_{2}^{\prime}$. Applying induction on $\operatorname{Max}\left(N, N^{\prime}\right)$, this shows that the asynchronous fellow traveller property is true with a constant $K=3$, with 2 units of $K$ coming from elementary moves and 1 unit of $K$ coming from a relabelling.

To synchronize the fellow traveller property and get a true automatic structure, the generating set and the language will be changed. For simplicity, we describe the changes only in the case where $S$ is a closed, oriented surface with one puncture. There are certain subwords of combing sequences of the form $\delta_{n} \rightarrow \cdots \rightarrow \delta_{n^{\prime}}$ such that $\delta_{n^{\prime}}$ and $\delta_{n}$ differ by a Dehn twist $\tau$ on a closed curve $\gamma$ which is a boundary component of a regular neighborhood of some $\operatorname{arc} h$ of $\delta_{n}$; these Dehn twists are described in [M1], and are used to make the computation of conjugacy invariants more efficient. The morphism $\left\{\delta_{n}, \delta_{n^{\prime}}\right\}$ is called a Dehn twist generator, and if $n<i<n^{\prime}$ then the morphism $\left\{\delta_{n}, \delta_{i}\right\}$ is called a fractional Dehn twist generator. The alphabet $A_{1}$ is taken to consist of all Dehn twist, fractional Dehn twist, and relabelling generators, and their inverses. Each word $w_{0}(\delta) \in L_{0}$ may be factored uniquely as a relabelling generator followed by a sequence of Dehn twist and fractional Dehn twist generators, with the proviso that no fractional Dehn twist generator can be absorbed into the previous Dehn twist factor. This factorization of $w_{0}(\delta)$ yields a word $w_{1}(\delta)$ over the alphabet $A_{1}$, and we take $L_{1}$ to be the set of all such words $w_{1}(\delta)$. The word acceptor for $L_{0}$ may, by slight modifications, be 
converted into a word acceptor for $L_{1}$, so $L_{1}$ is a regular language over $A_{1}$. The proof of the asynchronous fellow traveller property for $L_{0}$ may, with some effort, be souped up to prove the synchronous fellow traveller property for $L_{1}$, and we have:

Theorem. When $S$ is a closed, oriented surface with one puncture, the language $L_{1}$ is an automatic structure for $\operatorname{MCGD}(S)$, hence $\mathcal{M C G}(S)$ is automatic.

When $S$ is a general punctured surface, one must add additional words closely related to Dehn twists and the fractions thereof, in order to get a canonical factorization of words in $L_{0}$, and an automatic structure is obtained.

When $S$ is a closed surface, some new techniques are needed. Choosing a base point $x$ on $S$, one obtains a homomorphism $\mathcal{M C G}(S, x) \rightarrow \mathcal{M C G}(S)$ from the once-punctured mapping class group to the closed mapping class group. When $c$ is a closed curve on $S$ based at $x$, then by pushing the point $x$ around the curve $c$, one obtains an element of $\mathcal{M C G}(S, x)$ denoted push $_{c}$. This yields a homomorphism push: $\pi_{1}(S) \rightarrow \mathcal{M C G}(S, x)$, and when $\chi(S) \leq-2$ we obtain a short exact sequence as follows [B]:

$$
1 \rightarrow \pi_{1}(S, x) \rightarrow \mathcal{M C G}(S, x) \rightarrow \mathcal{M C G}(S) \rightarrow 1
$$

The cases when $\chi(S) \geq-1$ all yield mapping class groups which are wellknown automatic groups.

In [M4], we consider a short exact sequence of finitely generated groups

$$
1 \rightarrow K \rightarrow G \rightarrow H \rightarrow 1
$$

such that $K$ is non-elementary word hyperbolic. In this situation, we prove that many geometric properties of the group $G$ are inherited by its quotient $H$. This is true in particular of the property of "combability". Recall that a finitely generated group $G$ is combable if there exists a (bounded synchronous) combing consisting of a surjective language $L$ of normal forms satisfying the fellow traveller property. Thus, an automatic structure is a regular combing. If $L$ is a combing of $G$ and $K$ is non-elementary word hyperbolic, a combing for $H$ is defined as follows. Let $\mathcal{T}$ be the space of distinct triples of points on the boundary of $K$. The automorphism group of $K$ acts on $\mathcal{T}$ by homeomorphisms, hence $G$ acts on $\mathcal{T}$ by homeomorphisms. Let the action be denoted $\mathcal{A}_{g}: \mathcal{T} \rightarrow \mathcal{T}$ for $g \in G$. Restricting this action to $K$ yields a properly discontinuous, cocompact action. Choose a compact fundamental domain $C \subset \mathcal{T}$ for the action of $K$, which means simply that 
the translates of $C$ by $K$ cover all of $\mathcal{T}$. Also, choose a base point $\xi \in \mathcal{T}$. With these choices, a sublanguage $L^{c} \subset L$ may be defined as follows:

$$
L^{c}=\left\{w \in L \mid \xi \in \mathcal{A}_{\bar{w}} C\right\}
$$

It is proved in [M4] that $L^{c}$ is a combing of $H$.

Since $\pi_{1}(S, x)$ is a non-elementary word hyperbolic group, and since $\mathcal{M C G}(S, x)$ is automatic, it follows that $\mathcal{M C G}(S)$ is combable. This already proves many of the geometric properties of automatic groups, such as the quadratic isoperimetric inequality. To obtain more, we must show that a combing for $\mathcal{M C G}(S)$ can be defined in a more strictly constructive manner, so that the combing is regular. This is done first by constructing a short exact sequence of groupoids

$$
1 \rightarrow \pi_{1}(S, p) \rightarrow \mathcal{M C G D}(S, p) \rightarrow \mathcal{M C G D}(S) \rightarrow 1
$$

where the associated group of the groupoid

$$
\operatorname{MCGD}(S)=\mathcal{M C G D}(S, p) / \pi_{1}(S, p)
$$

is $\mathcal{M C G}(S)$. The construction given above, using the space $\mathcal{T}$ of triples of points in the boundary of $\pi_{1}(S, p)$, may be generalized to the groupoid setting, hence we may define a sublanguage $L_{1}^{c} \subset L_{1}$ which is a combing of the groupoid $\mathcal{M C G D}(S)$. To do this, first one chooses a base point $\xi \in \mathcal{T}$. Then one chooses a fundamental domain system which associates, to each ideal triangulation $\delta$ of $(S, p)$, a fundamental domain $C_{\delta}$ for the action of $\pi_{1}(S, p)$ on $\mathcal{T}$, so that $C_{\delta}$ depends equivariantly on $\delta$, i.e. for each $\Phi \in \mathcal{M C G}(S, p), \mathcal{A}_{\Phi} C_{\delta}=C_{\Phi \delta}$. Then define

$$
L_{1}^{c}=\left\{w_{1}(\delta) \in L_{1} \mid \xi \in C_{\delta}\right\}
$$

The methods of [M4] are then adapted to prove that $L_{1}^{c}$ is a combing of $\mathcal{M C G D}(S)$. It remains to choose $\xi$ and the fundamental domain system $\left\{C_{\delta}\right\}$ carefully so that $L_{1}^{c}$ is a regular language.

This is accomplished by making sure that $C_{\delta}$ is "finitely defined". Intuitively, this means that for any point $\eta=\left(\eta_{1}, \eta_{2}, \eta_{3}\right) \in \mathcal{T}$, the decision about whether $\eta \in C_{\delta}$ can be made purely in terms of the interaction of the points $\eta_{1}, \eta_{2}, \eta_{3} \in \partial \pi_{1}(S, p)$ with the arcs of $\delta$, and with the arcs of triangulations which differ from $\delta$ by at most a constant number of elementary moves. Points in $\partial \pi_{1}(S, p)$ are compared with arcs of $\delta$ by lifting each arc to the universal cover of $S$, taking a connected lift passing through a base point $\tilde{p}$ lying over $p$, and using the endpoints at infinity of the lift. We show 
that $\mathcal{T}$ may be exhausted by a sequence of compact sets $C_{\delta, k}$, depending equivariantly on $\delta$, so that each $C_{\delta, k}$ is finitely defined. Since $\pi_{1}(S, p)$ acts properly discontinuously and cocompactly on $\mathcal{T}$, then for each $\delta$ there exists $k$ such that $C_{\delta, k}$ is a fundamental domain for $\mathcal{T}$. Choosing $k=k(\delta)$ minimally so that $C_{\delta}=C_{\delta, k(\delta)}$ is a fundamental domain, we obtain a fundamental domain system $\left\{C_{\delta}\right\}$ such that $C_{\delta}$ is finitely defined in terms of $\delta$. The base point $\xi$ is then chosen to be any "finitely defined" point of $\mathcal{T}$. Using this base point and fundamental domain system, carry out the construction of $L_{1}^{c}$ described above, obtaining a combing for $\mathcal{M C G D}(S)$. Using finite definability of $C_{\delta}$, together with the word acceptor automaton and the multiplier automata for the automatic structure $L_{1}$, it is then proved that $L_{1}^{c}$ is regular. Thus:

Theorem. When $S$ is a closed surface, the language $L_{1}^{c}$ is an automatic structure for $\mathcal{M C G D}(S)$, hence the group $\mathcal{M C G}(S)$ is automatic.

As a final remark, when $S$ is punctured the automatic structure can be efficiently computed in terms of the topological type of $S$. But when $S$ is closed we do not know how to efficiently find the automatic structure, because of our inability to find an efficient formula for the parameter $k(\delta)$ described above.

\section{References}

[B] J. Birman, Braids, Links, and Mapping Class Groups, Princeton University Press, 1975.

[ECHLPT] D. Epstein, J. Cannon, D. Holt, S. Levy, M. Paterson, W. Thurston, Word processing in groups, Jones \& Bartlett, 1992.

[H] A. Hatcher, On ideal triangulations of surfaces, Topology and its Applications 40 (1992), no. 2, 189-194.

[HV] A. Hatcher, K. Vogtmann, Isoperimetric inequalities for automorphisms groups of free groups, preprint (1993).

[M1] L. Mosher, Pseudo-Anosovs on Punctured Surfaces, Princeton University Dissertation, 1983.

[M2] , Tiling the projective foliation space of a punctured surface, Trans. AMS 306 (1988), 1-70.

[M3] _ Mapping class groups are automatic, preprint (1993).

[M4] Hyperbolic extensions of groups, preprint (1993).

[P] R. Penner with J. Harer, Combinatorics of Train Tracks, Princeton University Press, 1992.

\footnotetext{
Departiment of Mathematics and Computer Science, Rutgers Univerity, NEWARK, N J 07102

E-mail address: mosher@andromeda.rutgers.edu
} 